\title{
Umbilical artery to common femoral artery (CFA) transposition, a novel technique for limb salvage in a newborn: a case report
}

\author{
Hamidreza Hosseinpour ${ }^{1 \oplus}$, Ali Bahador ${ }^{2 \oplus}$, Ali Tadayon ${ }^{2 \odot}$ \\ ${ }^{1}$ Department of Surgery, Shiraz Laparoscopic Research Center, ${ }^{2}$ Department of Pediatric Surgery, Shiraz University of Medical \\ Sciences, Shiraz, Iran.
}

\begin{abstract}
Background. Cardiac catheterization is a course of action with a low rate of complication; however, the potential risk factors should be considered before the procedure. The risk of arterial complications increases in sick premature infants, especially in the first days of life.

Case. A four-day-old neonate with cyanotic heart disease (tricuspid atresia) was referred to our tertiary center for patent ductus arteriosus (PDA) stenting by cardiac catheterization. During catheterization, the stent escaped and was trapped in the left external iliac artery. Following the stent retrieval, the left external artery was disrupted entirely and caused pulse-less left lower extremity. The patient was immediately transferred to the operating room to repair the artery. Retracted ends of the artery had caused a 3-4 $\mathrm{cm}$ space between them. In this rare and emergency situation, the left umbilical artery was used to maintain the common femoral artery's (CFA) blood supply. Therefore, an end to end anastomosis of the distal part of the left umbilical artery with the proximal part of the left CFA was done successfully and uneventfully.

Conclusions. The complications of cardiac catheterization that lead to surgical repair are almost challenging, and adequate preoperative planning should be performed. In selective cases, the umbilical artery can be used to maintain the blood flow to the common femoral artery.
\end{abstract}

Keywords: cardiac catheterization, complication, surgical repair, newborn, rupture.

Congenital heart disease (CHD) is a progressive global problem in infants. Catheter-based intervention has improved the survival and quality of life. ${ }^{1}$ It affects nearly 800 per 100,000 births and has caused death in about $6 \%$ of US infants during the past decade. ${ }^{2}$

For patients with established CHD, cardiac catheterization procedures can offer less invasive interventional therapy, for patients with complex lesions. ${ }^{3}$ Although the overall rate of complications in catheterization procedures is very low, they can cause various

\footnotetext{
$凶$ Ali Tadayon

alitad4@gmail.com
}

Received 20th October 2020, revised 9th January 2021, accepted 11th February 2021. unintentional cardiac and vascular adverse events including cardiac arrest, prolonged ischemia, pseudo-aneurysm, and even vessel injury and perforation. ${ }^{4}$

One of the most infrequent but emergent complications of cardiac catheterization is stent loss, which can often be successfully handled percutaneously. ${ }^{5}$ However, our patient went through a complete disruption of the left external iliac artery during stent retrieval.

Following recognized disruption of the left external iliac artery, our patient underwent emergency exploration of the left femoral area and retro-peritoneum and successful repair of the artery by performing end to end anastomosis of the distal left umbilical artery to the proximal common femoral artery (CFA). 


\section{Case Report}

A 4-day-old female who weighed $2.3 \mathrm{~kg}$ and was the product of a normal vaginal delivery was referred to our pediatric cardiologist in a tertiary referral center due to cyanosis. On arrival, the patient was cyanotic, with oxygen saturation $\left(\mathrm{O}_{2}\right)$ of $60 \%$ at room air that increased to $65-68 \%$ with oxygen therapy. The heart rate was $160 \mathrm{bpm}$ with a respiratory rate of 66 per minute and a blood pressure of 70/45 $\mathrm{mmHg}$. She was mildly tachypneic with a single S2 and a 3/6 pansystolic murmur in the left subclavicular area, and there was no evidence of hepatomegaly. The cardiac axis was $60^{\circ}$, and the chest radiography showed decreased pulmonary vascularity.

She was admitted to our unit for echocardiography and further evaluation. Bedside transthoracic echocardiography revealed tricuspid atresia, pulmonary atresia, hypoplastic right ventricle (RV), mediumsized atrial septal defect (ASD), medium-size patent ductus arteriosus (PDA), very small (pinhole) size ventricular septal defect (VSD), good left ventricular (LV) systolic function, thickened mitral valve, and severe pulmonary hypertension.

After primary stabilization (hydration, correcting acidosis, preparing packed red blood cell (RBC), and starting prostaglandin $\mathrm{E}_{1}$ with a dose of $0.05 \mu \mathrm{g} / \mathrm{kg} / \mathrm{min}$ ), the patient was transferred to catheterization laboratory for cardiac catheterization for PDA stenting and atrial septostomy. Prostaglandin was discontinued three hours prior to the procedure. Under general anesthesia, multiple attempts were made for the left axillary artery access, but they all failed due to tortuous PDA, and our cardiologist was unable to get into the PDA through the left axillary artery. Meanwhile, intravascular heparin was started for him with a dose of $50 \mathrm{U} / \mathrm{kg}$. The right axillary artery was then cannulated for PDA stenting under fluoroscopy guidance, using a Rontis Coronary stent system (Rontis AG, Europa Ultra, Switzerland, $4^{*} 20 \mathrm{~mm}$ ). The stent was dislodged to the left external iliac artery (probably due to the short length and relatively large (3.5 $\mathrm{mm}$ ) diameter of PDA. For retrieving the lost stent, the left femoral artery was accessed, and, after multiple attempts, the stent was removed via an introducer and a $4 \mathrm{~mm}$ snare. After the procedure, aortogram failed to show any flow passing through the left iliac artery (Fig. 1). Doppler ultrasound (DUS) of the left lower extremity confirmed the loss of color flow from the femoral artery up to the posterior tibialis. Pediatric surgery consultation was done, and the patient was immediately transferred to the operating room for iliac artery repair.

In supine position, the patient was prepped and draped in the usual sterile surgical fashion. Under general anesthesia, a vertical incision was made from the left lower quadrant to the inguinal canal and proximal part of the thigh. Exploration of the left external iliac, left CFA and vein was done. The left external iliac artery was utterly disrupted. At first sight, the distal end was present, but the proximal end was not found. Thus, we extended the incision

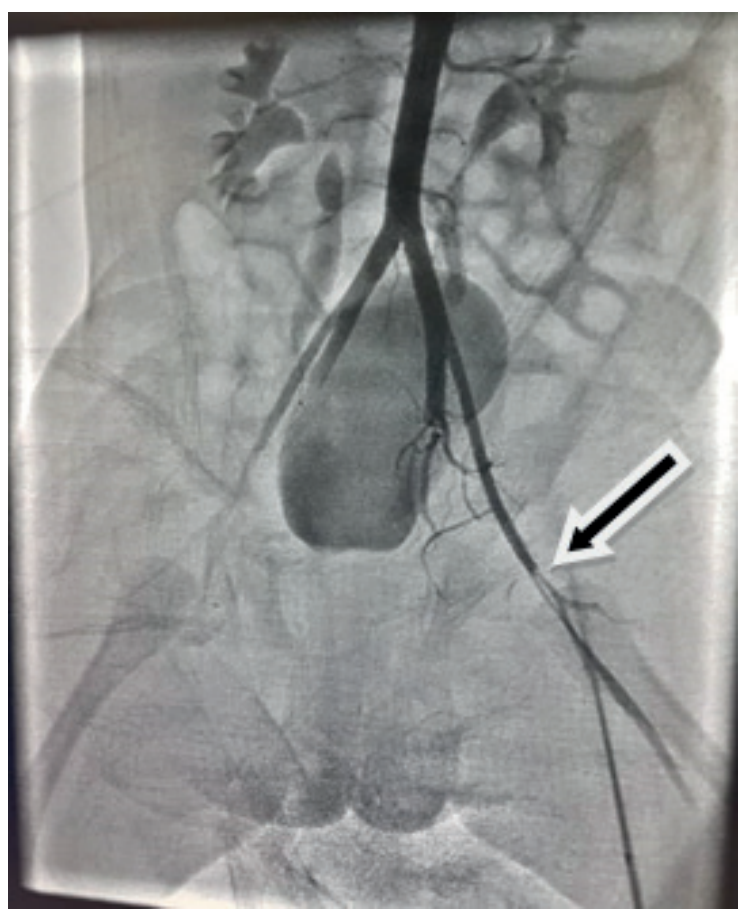

Fig. 1. Abnormal angiography of the left femoral artery 
vertically and then the proximal end, which was retracted for about $4-5 \mathrm{~cm}$, was seen in the retroperitoneum. At first, we tried to anastomose the two ends of the artery with each other, but the vessels were under tension that led to narrowing of the lumen and increased risk of future thrombosis. In this emergent situation, we had to find a way to maintain the CFA's blood supply to prevent left lower extremity ischemia. There were some potentially impossible options like reverse saphenous vein graft anastomosis that all could cause more severe complications due to vein diameter at this age.

During our exploration, we incidentally found the end of the left umbilical artery, which was pulsatile, so the idea of using the umbilical artery as the supplier of CFA came to our mind. The left umbilical artery was incised from its end near the umbilicus. There was no blood flow, so Fogarty catheterization (NO 3.5) was performed for embolectomy, which was effective with a good jet flow. Then, we put a bulldog vascular clamp at the end of the umbilical artery and tried to perform Fogarty catheterization of the proximal end of CFA until the back-flow perfusion appeared. At this time, the umbilical artery was rotated downward and brought down near the proximal end of CFA, and following spatulating the smaller vessel, an end to end anastomosis was done by simple interrupted sutures with prolene 8-0 under loupe magnification. The distal end of the external iliac artery was ligated with silk 3-0. The femoral artery had a good flow with a satisfying pulse. Figure 2 shows the left external iliac artery after anastomosis. After homeostasis, the fascia was closed with PDS 3-0, and the skin was closed with nylon 3-0. Anti-coagulation therapy was started for her immediately after the procedure with intravascular administration of heparin every 12 hours.

The postoperative course was uneventful, and the patient was transferred to neonatal intensive care unit (NICU) for medical therapy and stabilization before another attempt for a percutaneous procedure or a modified BlalockTaussig shunt. Following the next 48 hours, the common femoral, popliteal and posterior pedal pulses were bilaterally and symmetrically present, and no sign of ischemia or bleeding was present. Unfortunately, the patient eventually died due to her cardiac conditions and clearly unrelated to her last procedure.

Consent was obtained from the parents regarding the publication of this case report.

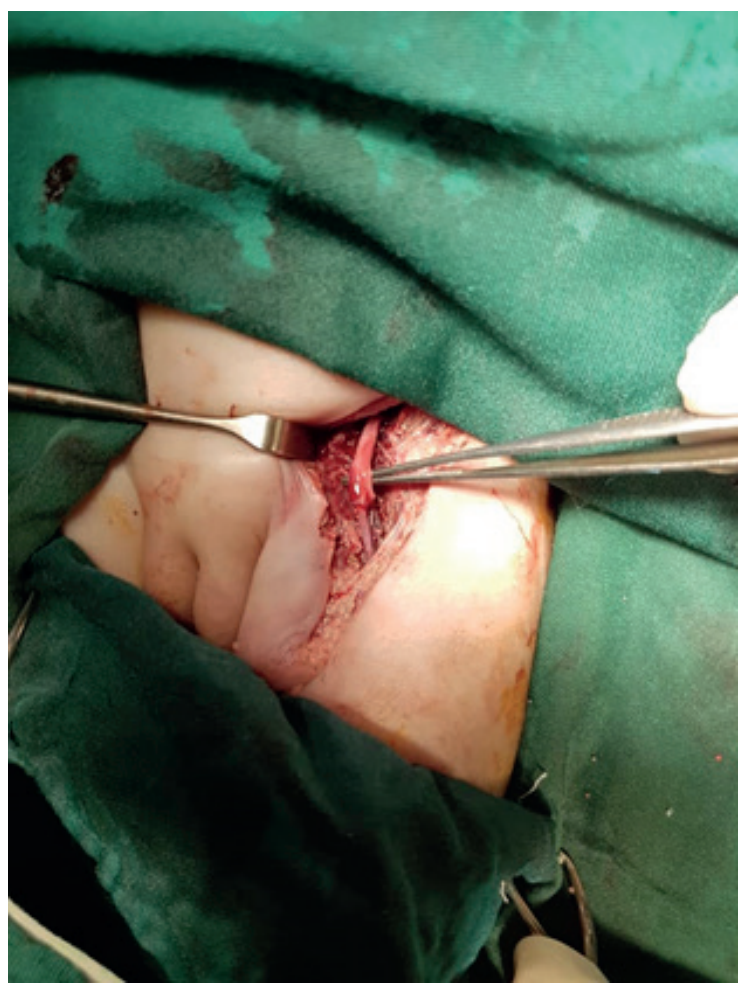

Fig. 2. Repaired left external iliac artery after the procedure

\section{Discussion}

Angiography is a well-known diagnostic and interventional procedure that is used worldwide with well-established low rate of complications. According to a study conducted by Hessel et al. ${ }^{6}$, complications originating from the accesssite of catheterization are the most common ones, and generally among transfemoral and transaxillary approaches, the transfemoral technique is the method of choice because it is both easier and safer. In another study, it is claimed that the overall rate of major vascular complications is about $4.85 \%{ }^{7}$ 
In a large single-center experience, conducted by Brilakis et al. ${ }^{8}$, it was revealed that stent loss, as a stressful complication, still occurs with an incidence of $0.32 \%$ in general. Their results revealed that there was an $86 \%$ rate of successful retrieval, and $26 \%$ of them were done by snare loop. Among all, 11,773 patients who participated in this study and 38 patients who went through stent loss as a complication, in only three patients, the stent was subsequently immobilized in the femoral arteries, which was retrieved by small-balloon and two-wire technique. Although facing this stressful experience is somehow difficult, our pediatric cardiology team tried to retrieve the stent by snare loop immediately, but it caused subsequent complications afterward.

The best approach to a susceptible artery injury is a complete, detailed vascular examination to recognize any changes which result in a pale and pulseless extremity. ${ }^{9}$ This is what our pediatric cardiology team had done in the Cath lab. As soon as they noticed the absence of palpable pulse in the left lower extremity, and while the aortogram failed to show any flow in the left iliac artery, DUS was done and the report confirmed the diagnosis.

A study by McMillan et al. ${ }^{10}$ noted that surgical repair post-arterial injury in neonates is unlikely (less than $0.4 \%$ ), and if surgical intervention is required, it is probably complex and necessitates a vascular graft. The main problem with our case was the distance between the two ends of the disrupted artery, which was about 3-4 cm. That means there was no choice except using vascular autograft, which was impossible due to the emergency situation and age of the neonate; that is when the idea of using umbilical artery occurred.

There were some graft options in this situation. First, we could ligate the artery to stop the bleeding and hope for collateral arteries to supply the left lower limb ${ }^{11}$, which was impossible, as the limb was going cold and cyanotic. The next option was harvesting the jugular vein as an autograft ${ }^{12}$, a very sophisticated procedure, as the patient was a neonate with a relatively short neck. One of the controversial options was the utilization of the contralateral saphenous or femoral vein, again as an autograft $\mathrm{fl}^{13}$, which was also not feasible due to the procedure's time-consuming nature. So, using the umbilical artery was a promising way of maintaining the limb's blood supply, at least transiently.

At the umbilicus, the umbilical vessels enter the abdomen. The two umbilical arteries are then extended as the hypogastric arteries and moved to the back of the anterior abdominal wall and the sides of the bladder to their origin from the internal iliac arteries. The anatomy of the arteries is shown in Figure 3. With age, the parts of the hypogastric arteries continuing between the sides of the bladder and the umbilicus are eliminated, and they develop as fibrous cords, the lateral umbilical ligaments. The umbilical vein and ductus venosus are eliminated; the vein turns into the ligamentum teres, while the ductus develops into the ligamentum venosum of the liver. ${ }^{14-16}$

Although there are some other options (such as using femoral vein or intra-arterial shunt) for management of iliac artery disruption, this procedure worked as a bridge operation, which

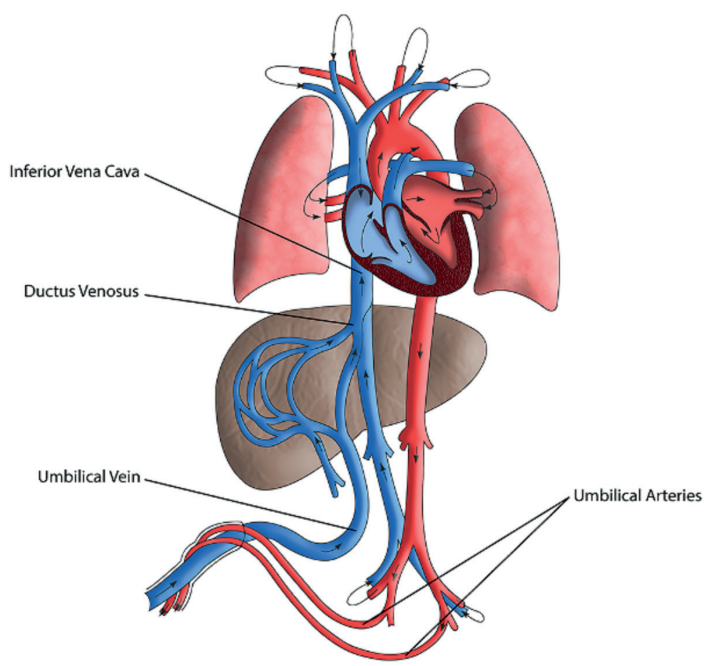

Fig. 3. Anatomy of the umbilical arteries and veins 
provided enough time to save the limb and keep the extremity warm until the patient got stable.

Cardiac catheterization is a course of action with a low rate of complications; however, potential risk factors should be considered before the procedure. On the other hand, complications that lead to surgical repair are almost always challenging and adequate preoperative planning should not be performed. Hence, in selective cases, the umbilical artery can be used to maintain the blood flow to the common femoral artery.

\section{Acknowledgements}

The authors would like to thank Shiraz University of Medical Sciences, Shiraz, Iran and also Center for Development of Clinical Research of Nemazee Hospital and Dr. Nasrin Shokrpour for editorial assistance.

\section{Author contribution}

The authors confirm contribution to the paper as follows: study conception and design: $A B$, AT; data collection: $\mathrm{HH}$; draft manuscript preparation: HH. All authors reviewed the results and approved the final version of the manuscript.

\section{Conflict interest}

The authors declare that they have no competing interests.

\section{REFERENCES}

1. Zimmerman MS, Smith AGC, Sable CA, et al; GBD 2017 Congenital Heart Disease Collaborators. Global, regional, and national burden of congenital heart disease, 1990-2017: a systematic analysis for the Global Burden of Disease Study 2017. Lancet Child Adolesc Health 2020; 4: 185-200.

2. Abouk R, Grosse SD, Ailes EC, Oster ME. Association of US state implementation of newborn screening policies for critical congenital heart disease with early infant cardiac deaths. JAMA 2017; 318: 21112118.
3. Gao Y, Wang P, Su Y, et al. Cardiac catheterization procedures in children with congenital heart disease: increased chromosomal aberrations in peripheral lymphocytes. Mutat Res 2020; 852: 503163.

4. Tokel K, Gümüş A, Ayabakan C, Varan B, Erdoğan İ. Complications of cardiac catheterization in children with congenital heart disease. Turk J Pediatr 2018; 60: 675-683.

5. Iturbe JM, Abdel-Karim AR, Papayannis A, et al. Frequency, treatment, and consequences of device loss and entrapment in contemporary percutaneous coronary interventions. J Invasive Cardiol 2012; 24: 215-221.

6. Hessel SJ, Adams DF, Abrams HL. Complications of angiography. Radiology 1981; 138: 273-281.

7. Kugelmass AD, Cohen DJ, Brown PP, Simon AW, Becker ER, Culler SD. Hospital resources consumed in treating complications associated with percutaneous coronary interventions. Am J Cardiol 2006; 97: 322-327.

8. Brilakis ES, Best PJ, Elesber AA, et al. Incidence, retrieval methods, and outcomes of stent loss during percutaneous coronary intervention: a large singlecenter experience. Catheter Cardiovasc Interv 2005; 66: 333-340.

9. McCann RL, Schwartz LB, Pieper KS. Vascular complications of cardiac catheterization. J Vasc Surg 1991; 14: 375-381.

10. McMillan I, Murie JA. Vascular injury following cardiac catheterization. Br J Plast Surg 1984; 71: 832835.

11. Mays ET, Wheeler CS. Demonstration of collateral arterial flow after interruption of hepatic arteries in man. New Engl J Med 1974; 290: 993-996.

12. Holsworth IG, Kyles AE, Bailiff NL, Hopper K, Long C, Ilkiw JE. Use of a jugular vein autograft for reconstruction of the cranial vena cava in a dog with invasive thymoma and cranial vena cava syndrome. J Am Vet Med Assoc 2004; 225: 1205-1210.

13. Perry MO, Thal ER, Shires GT. Management of arterial injuries. AnnSurg 1971; 173: 403-408.

14. Emmanouilides GC, Rein BI. Abdominal aortography via the umbilical artery in a newborn infant. Radiology 1964; 82: 447-4450.

15. Gray H, Lewis WH. Anatomy of the Human Body. (20th ed) Philadelphia: Lea \& Febiger, 1918.

16. Paster SB, Middleton P. Roentgenographic evaluation of umbilical artery and vein catheters. JAMA 1975; 231: 742-746. 